\title{
Programmed Cell Death in SARS-CoV-2 Infection: A Short Review
}

\author{
Rushikesh Deshpande ${ }^{1}$ and Chunbin Zou ${ }^{1,2, * \text { (D) }}$ \\ 1 Department of Environmental and Occupational Health, Graduate School of Public Health, \\ University of Pittsburgh, Pittsburgh, PA 15213, USA; RHD10@pitt.edu \\ 2 Division of Pulmonary, Allergy, Critical Care Medicine, Department of Medicine, School of Medicine, \\ University of Pittsburgh, Pittsburgh, PA 15213, USA \\ * Correspondence: zouc@upmc.edu; Tel.: +1-(412)-624-4404
}

check for updates

Citation: Deshpande, R.; Zou, C. Programmed Cell Death in SARS-CoV-2 Infection: A Short Review. J. Respir. 2021, 1, 223-228. https://doi.org/10.3390/jor1040021

Academic Editor: Cesar A. Moran

Received: 16 August 2021

Accepted: 21 September 2021

Published: 24 September 2021

Publisher's Note: MDPI stays neutral with regard to jurisdictional claims in published maps and institutional affiliations.

Copyright: (c) 2021 by the authors. Licensee MDPI, Basel, Switzerland. This article is an open access article distributed under the terms and conditions of the Creative Commons Attribution (CC BY) license (https:// creativecommons.org/licenses/by/ $4.0 /)$.

\begin{abstract}
Severe acute respiratory syndrome coronavirus 2 (SARS-CoV-2), the latest variant in the coronavirus family, causing COVID-19, has resulted in global pandemic since early 2020 leading to severe public health concern. So far, the pandemic has caused more than 200 million infections and 4 million deaths worldwide. Most of the studies are focused on developing prevention, intervention, and therapeutic strategies. However, underlying pathophysiology of the disease is important as well, which needs further attention. Cell death is one of the major causative mechanisms that leads to severe inflammation, and it is also an a posteriori consequence of the hyperinflammatory storm that renders poor prognosis of the disease. Substantial cell death has been reported in biopsy samples from post mortem patients. Among the distinct cell death pathways, apoptosis, the regulated programmed cell death plays an important role in the pathogenesis of the disease. Understanding the role of SARS-CoV-2 infection in apoptosis is critical to linearize the pathogenesis of the virus as well as the resultant disease, that may uncover novel therapeutic targets in treatment of COVID19 patients. Here, we review the current progress on the underlying molecular mechanism(s) of SARS-CoV-2-induced apoptosis, not only at the level of the virus but also at its individual proteins.
\end{abstract}

Keywords: severe acute respiratory syndrome coronavirus 2 (SARS-CoV-2); apoptosis; ORF3a; ORF6a; NSP6; ORF7a; ORF8

\section{Introduction}

Severe acute respiratory syndrome coronavirus 2 (SARS-CoV-2) is a novel coronavirus responsible for the coronavirus disease 2019 (COVID-19), which has led to a global pandemic resulting in over 200 million infections and 4.6 million deaths thus far (source: Johns Hopkins University, 10 September 2021). Coronavirus is a group of viruses belonging to the family Coronaviridae of order Nidovirales [1]. Among the family, seven of them are known to infect humans, namely, Human coronavirus HKU1 (HCoV-HKU1), NL63 Human coronavirus NL63 (HCoV-NL63), OC43 Human coronavirus OC43 (HCoV-OC43), 229E Human coro-navirus 229E (HCoV-229E), Severe Acute Respiratory Syndrome-CoV (SARS-CoV), Middle East respiratory syndrome-CoV (MERS-CoV), and SARS-CoV-2 [2]. SARS-CoV-2 is the seventh coronavirus identified recently in 2020 with high genetic similarity with SARS-CoV. Clinically, HKU1, NL63, OC43, and 229E cause mild symptoms, whereas SARS$\mathrm{CoV}, \mathrm{MERS}-\mathrm{CoV}$, and SARS-CoV-2 are highly transmissible that can lead to severe diseases and death [3]. SARS-CoV caused the first pandemic in 2002 since the beginning of the 21st century. MERS-CoV led to pandemic in 2012. The most recent SARS-CoV-2-induced COVID-19 pandemic has severely impaired public health and economy all over the world.

SARS-CoV-2 virus has a single stranded and positive-sense RNA which is enveloped with glycoproteins. The length of the SARS-CoV-2 genome is about 30,000 base-pairs [4]. There are 28 confirmed proteins encoded by the SARS-CoV-2 genome, with polyproteins ORF1ab and ORF1a which form part of the $5^{\prime}$-end of the genome, cleaved into 16 different 
non-structural proteins (NSP), NSP 1 to 16 , while the $3^{\prime}$-end of the genome comprising 4 structural proteins- spike $(\mathrm{S})$, membrane $(\mathrm{M})$, nucleocapsid $(\mathrm{N})$, and envelope $(\mathrm{E})$, as well as 8 accessory proteins-Open Reading Frames (ORFs) 3a, 3b, 6, 7a, 7b, 8, 9b, and 10 [4]. Spike protein is known to interact with human angiotensin converting enzyme 2 (ACE2) that grants the virus access to host cells [4]. NSP proteins are believed to function in viral replication, assembly, and secretion [4]. The accessory ORF proteins are responsible for countering the host defense and immunity to facilitate viral infection [4]. Much of the focus so far has been on the mitigating as well as therapeutic intervention strategies against the virus, but it is also equally important to expand our knowledge in understanding how the virus operates at cellular level as well as the role of its individual proteins.

SARS-CoV-2 infection results in diversified inflammatory responses from host cells, and one such response is the host cell death. Cell death is an important process required to maintain homeostasis as well as a healthy environment for functioning cells, involving the removal of infected or damaged cells. Understanding the role of SARS-CoV-2 as well as its individual encoded proteins in cell death is a crucial aspect for better comprehending the pathogenesis of the disease. Cell death has multiple mechanistic classifications, with apoptosis, necrosis, and pyroptosis being the three important ones in anti-viral response [5]. However, for the scope of this review, we are going to focus on apoptosis and the role SARS-CoV-2 plays in apoptosis of host cells.

\section{Apoptosis}

Apoptosis is a highly regulated programmed cell death, playing an important role in determining the pathological outcome based on the way it is activated [6]. It can be initiated for inhibiting the virus propagation, however, it can also cause lung damage if it becomes excessive [7]. Uncontrolled apoptosis is a symptom of certain diseases, like Alzheimer's, while inhibited apoptosis is the main feature of others, like cancer [8]. In viral infection, apoptosis is promoted for releasing viral particles [9]. There are several mechanisms enveloped viruses employ, such as the use of death receptor orthologs, to counter the apoptosis promoted by host cells [9]. As a host defense, prompt apoptosis may reduce viral replication and limit lesion in infected tissues. Apoptosis can be initiated from both extrinsic and intrinsic apoptotic pathways [10]. The extrinsic pathway, also called the death receptor pathway, involves the use of death receptors belonging to the family of the tumor necrosis factor receptor genes [11]. TNF-R1 (tumor necrosis factor receptor-1) and FAS/CD95 (FS-7-associated surface antigen/cluster of differentiation 95) are the two important members of the extrinsic pathway. TNFR1 and FAS/CD95 interact with receptors and help recruit Fas-associated death domain protein (FADD) as well as pro-caspase 8 , for assembling death-inducing signaling complex (DISC) [12]. FADD in the DISC cleaves caspase-8, which in turn activates caspases 6 and 7, leading to the subsequent activation of caspase 3 , which finally culminates downstream signal transduction and gnomic DNA cleavage [12]. Apart from this, DR4/DR5 also play a role by binding to TNF-related apoptosis-inducing ligand (TRAIL) receptors and causing TRAIL-induced IkB kinase (IKK) activation [12]. The intrinsic pathway also called the mitochondrial pathway, and is activated due to external stimuli such as DNA damage or cell stress [13]. The external stimuli trigger Bak/Bax (BCL2 antagonist killer/BCL2 associated X protein), resulting in the release of cytochrome $C$ from mitochondria, thereby activating caspase 9 [13]. Activated caspase 9 then causes the cleavage of caspase 3, resulting in cell death [13].

\section{SARS-CoV-2 Induces Lung Epithelial Cell Apoptosis}

The infection of SARS-CoV-2 in lung epithelial cells initiates cell death as well as inflammatory response [14]. The cell death is caused by the activation of caspase 8 . TUNEL (Terminal deoxynucleotidyl transferase dUTP nick end labeling) assay was performed on human lung cancer epithelial Calu-3 cells infected with the virus to determine if apoptosis was induced [14]. Comparison of TUNEL staining of mock cells with infected cells revealed that the infected cell group had a relatively higher number of apoptotic cells [14]. 
Western blot analysis indicated that caspases 8,9, and 3 were activated in the infected cells. Additionally, activation of PARP-1 (poly (ADP-ribose) polymerase-1) was also observed in virus-infected cells [14]. When the cells were treated with caspase-8 inhibitor named Z-IETD-FMK, it resulted in the inhibition of caspases 3 activation as well as the cleavage of BID (BH3 Interacting Domain Death Agonist) and PARP-1 [14]. Thus, the results suggesting the involvement of caspase 8 dependent apoptosis strengthens the hypothesis that caspase 8 plays a pivotal role in SARS-Cov-2 based apoptosis as well as inflammatory response.

A more recent study which analyzed the post-mortem lung pathologies of COVID-19 patients, observed significant increase in apoptotic signals, the signals being observed in microvascular endothelial cells (ECs) and vascular intima [15]. This study used TUNELImmunofluorescence double labeling assay and found that both extrinsic as well as intrinsic apoptotic pathways were involved due to SARS-CoV-2 infection. It was also observed that pharmacological activators of Epac1 protected endothelial cells in co-cultures of HUVECs (human umbilical vein endothelial cells) and Vero cells after infection with the virus.

A latest study published further consolidated the critical role of apoptosis in the pathogenesis of COVID-19 [16]. The authors used MERS-CoV as a model to understand the underlying mechanism for the apoptosis caused by the virus, and verified SARSCoV-2-induced apoptosis by combining in vitro, in vivo, as well as ex vivo models [16]. They identified that PERK (protein kinase R-like endoplasmic reticulum kinase) signaling played a central role in regulating proapoptotic mediators in the MERS-CoV infection [16]. PERK activation occurred due to its displacement from GRP78 (78 KDa Glucose-Regulated Protein), an ER (Endoplasmic Reticulum) marker [16]. The PERK signaling was also observed to converge with the intrinsic or mitochondrial apoptotic pathway [16]. Inhibition of PERK signaling as well as the intrinsic apoptotic pathway led to the pathogenesis of both MERS-CoV as well as SARS-CoV-2 [16]. Modulation of PERK signaling ameliorated lung damage caused by SARS-CoV-2 infection [16]. These observations therefore not only provide evidence of the crucial role of apoptosis in the SARS-CoV-2 pathogenesis, but also gives an intervention strategy of targeting apoptosis for treating SARS-CoV-2.

\section{The Role of Individual SARS-CoV-2 Proteins in Apoptosis}

Our understanding of the role (s) of individual protein encoded by the virus genome in cell death and apoptosis is limited. However, there are few studies so far which elucidate the role of several proteins in cell death.

\section{ORF3a}

ORF3a, an accessory protein expressed by both SARS-CoV and SARS-CoV-2 has been identified to induce apoptosis [17]. SARS-CoV-2 causes apoptosis via ORF3a as well in distinct cell lines, including HEK293T (human embryonic kidney cell line), HepG2 (human liver cancer cell line), and Vero E6 (kidney epithelial cell line) cells [18]. Annexin V staining was used to determine if apoptosis was induced in the cells. Additionally, activated caspase 3 was examined using flow cytometry. Significantly higher number of cells had activated caspase 3 in the presence of ORF3a. The mechanism of the apoptosis was determined to be caspase- 8 dependent. Caspase 8 specifically cleaves Bid to cause mitochondrial cytochrome c release, thereby resulting in intrinsic apoptosis.

\section{ORF6, NSP6, and ORF7A}

A recent study observed that Orf6, NSP6, and Orf7a induce high levels of cytotoxicity in human 293T cells [19]. ORF6, ORF7a, and NSP6 proteins were mainly localized at the membrane, i.e., endoplasmic reticulum, autophagosome, and lysosomal membranes in COS-7 cells. ORF6 protein was not found at endosomes, but rather at lysosomes and autophagosomes. Proteomics revealed that ORF6 interacts with nucleopore proteins, namely RAE1, XPO1, and RANBP2. Inhibition of XPO1 pathway using an FDA-approved inhibitor Selinexor attenuated ORF6-induced cytotoxicity. Mitochondrial damage was 
observed in the muscle of ORFs 6, 7a, and NSP6 transgenic flies, suggesting that apoptosis might be the major pathway in ORF6, ORF7a, and NSP6 induced cytotoxicity [20].

\section{ORF8}

ORF8 from SARS-CoV induces apoptosis [21]. For SARS-CoV-2, it is reported that ORF8 induces ER stress via activation of IRE1 (Inositol-Requiring Enzyme 1) and ATF6 (Activating Transcription Factor 6) pathways [22]. ORF8 also acts as an interferon antagonist and limits the IFN- $\beta$ production [22]. Both ORF8L (Open Reading Frame 8 Leucine) and ORF8S (Open Reading Frame 8 Serine), two isoforms of SARS-CoV-2 ORF8 are distinct from each other by having leucine and serine, respectively, at amino acid 84 [23], activated ATF6 and IRE1 pathways, thereby inducing ER stress [22,23]. Interestingly, a PERK pathway which is also involved in ER stress induction, was not activated in SARSCoV-2 infection [22]. ORF8 reduced nuclear translocation of interferon regulatory factor 3 (IRF3) was inhibited interferon-stimulated genes 15 and 56 (ISG15 and ISG56), thereby antagonizing INF- $\beta$ production in HEK-293 T cells [22].

\section{Conclusions}

In this review we have summarized the latest understanding about SARS-CoV-2 induced apoptosis (Figure 1). Apoptosis indeed plays a central role in the pathogenesis of the COVID-19 disease. It is initiated by activation of caspase 8 [14]. PERK signaling plays a role along with the intrinsic pathway [16]. However, although, few studies have reported the role of individual proteins such as ORF3a, ORF6, ORF8, and NSP6 in causing apoptosis [18-22], the role of other individual proteins in this regard has still not been fully explored. In addition, the role of apoptosis in the pathogenesis of the disease needs further investigation. Furthermore, it is not clear if SARS-CoV-2 induces cell death via mechanism (s) other than apoptosis. In-depth understanding of SARS-CoV-2 induced cell death will help not only in developing more efficient intervention strategies but also in treating more toxic variants and the resultant pathology of the virus.

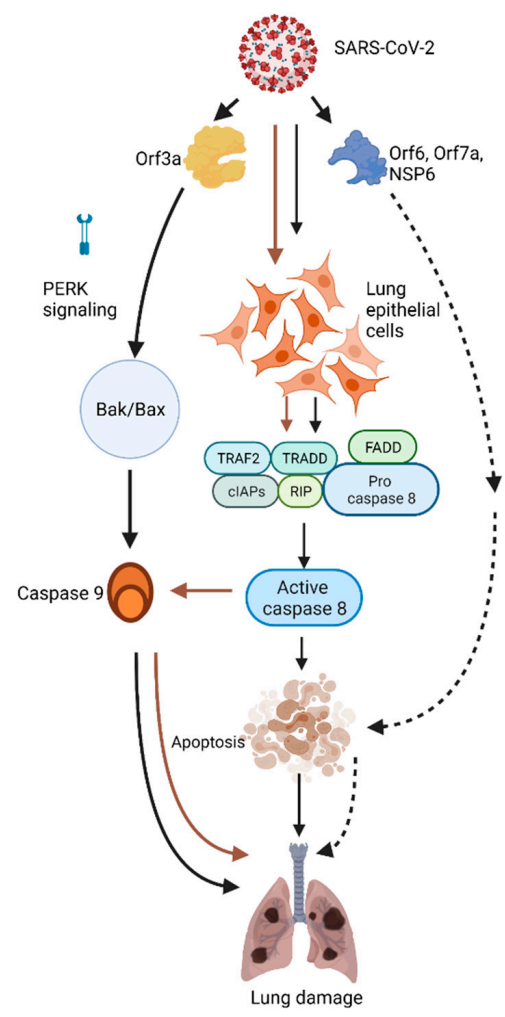

Figure 1. Representative schema describing the different apoptotic pathways associated with SARS CoV-2 and its encoded proteins in lung epithelial cells. The black arrows show the pathways 
associated with the virus-induced apoptosis, the brown arrows denote the pathway associated with ORF3a induced apoptosis while the dotted arrow indicates the pathway associated with the proteins ORF6, ORF7a, and NSP6, all localized at the membrane. However, ORF6 can translocate to lysosomes and autophagosomes. Dotted arrows are used for proteins and their speculative pathways. Figure created using biorender.com (10 September 2021).

Funding: This work is supported by the National Institute of Heart, Lung and Blood grant R01 HL142997 at The United States to C.Z.

Institutional Review Board Statement: Not applicable.

Informed Consent Statement: Not applicable.

Data Availability Statement: Not applicable.

Conflicts of Interest: The authors declare no conflict of interest.

\section{References}

1. Pal, M.; Berhanu, G.; Desalegn, C.; Kandi, V. Severe Acute Respiratory Syndrome Coronavirus-2 (SARS-CoV-2): An Update. Cureus 2020, 12, e7423. [CrossRef] [PubMed]

2. Liu, D.X.; Liang, J.Q.; Fung, T.S. Human Coronavirus-229E, -OC43, -NL63, and-HKU1 (Coronaviridae). Encycl. Virol. 2021, 2, $428-440$.

3. Ye, Z.W.; Yuan, S.; Yuen, K.S.; Fung, S.Y.; Chan, C.P.; Jin, D.Y. Zoonotic origins of human coronaviruses. Int. J. Biol. Sci. 2020, 16, 1686-1697. [CrossRef]

4. Yoshimoto, F.K. The Proteins of Severe Acute Respiratory Syndrome Coronavirus-2 (SARS CoV-2 or n-COV19), the Cause of COVID-19. Protein J. 2020, 39, 198-216. [CrossRef] [PubMed]

5. Galluzzi, L.; Vitale, I.; Aaronson, S.A.; Abrams, J.M.; Adam, D.; Agostinis, P.; Alnemri, E.S.; Altucci, L.; Amelio, I.; Andrews, D.W.; et al. Molecular mechanisms of cell death: Recommendations of the Nomenclature Committee on Cell Death 2018. Cell Death Differ. 2018, 25, 486-541.

6. Kennedy, P.G. Viruses, apoptosis, and neuroinflammation-A double-edged sword. J. Neurovirol. 2015, 21, 1-7. [CrossRef]

7. Barber, G. Host defense, viruses and apoptosis. Cell Death Differ. 2001, 8, 113-126. [CrossRef]

8. Fleisher, T.A. Apoptosis. Ann. Allergy Asthma Immunol. 1997, 78, 245-250. [CrossRef]

9. Zhou, X.; Jiang, W.; Liu, Z.; Liu, S.; Liang, X. Virus Infection and Death Receptor-Mediated Apoptosis. Viruses 2017, 9, 316. [CrossRef]

10. Hengartner, M.O. The biochemistry of apoptosis. Nature 2000, 407, 770-776. [CrossRef]

11. Ashkenazi, A.; Dixit, V.M. Death receptors: Signaling and modulation. Science 1998, 281, 1305-1308. [CrossRef]

12. Pereira, W.O.; Amarante-Mendes, G.P. Apoptosis: A programme of cell death or cell disposal? Scand. J. Immunol. 2011, 73, 401-407. [CrossRef] [PubMed]

13. Troiano, L.; Ferraresi, R.; Lugli, E.; Nemes, E.; Roat, E.; Nasi, M.; Pinti, M.; Cossarizza, A. Multiparametric analysis of cells with different mitochondrial membrane potential during apoptosis by polychromatic flow cytometry. Nat. Protoc. 2007, 2, 2719-2727. [CrossRef] [PubMed]

14. Li, S.; Zhang, Y.; Guan, Z.; Li, H.; Ye, M.; Chen, X.; Shen, J.; Zhou, Y.; Shi, Z.-L.; Zhou, P.; et al. SARS-CoV-2 triggers inflammatory responses and cell death through caspase-8 activation. Sig. Transduct. Target Ther. 2020, 5, 235. [CrossRef]

15. Liu, Y.; Garron, T.M.; Chang, Q.; Su, Z.; Zhou, C.; Qiu, Y.; Gong, E.C.; Zheng, J.; Yin, Y.W.; Ksiazek, T.; et al. Cell-Type Apoptosis in Lung during SARS-CoV-2 Infection. Pathogens 2021, 10, 509. [CrossRef]

16. Chu, H.; Shuai, H.; Hou, Y.; Zhang, X.; Wen, L.; Huang, X.; Hu, B.; Yang, D.; Wang, Y.; Yoon, C.; et al. Targeting highly pathogenic coronavirus-induced apoptosis reduces viral pathogenesis and disease severity. Sci. Adv. 2021, 7, eabf8577. [CrossRef]

17. Ren, Y.; Shu, T.; Wu, D.; Mu, J.; Wang, C.; Huang, M.; Han, Y.; Zhang, X.-Y.; Zhou, W.; Qiu, Y.; et al. The ORF3a protein of SARS-CoV-2 induces apoptosis in cells. Cell Mol. Immunol. 2020, 17, 881-883. [CrossRef]

18. Law, P.T.W.; Wong, C.H.; Au, T.C.C.; Chuck, C.-P.; Kong, S.-K.; Chan, P.; To, K.-F.; Lo, A.; Chan, J.; Suen, Y.-K.; et al. The 3a protein of severe acute respiratory syndrome-associated coronavirus induces apoptosis in Vero E6 cells. J. Gen. Virol. 2005, 86, 1921-1930. [CrossRef]

19. Lee, J.-G.; Huang, W.; Lee, H.; van de Leemput, J.; Kane, M.A.; Han, Z. Characterization of SARS-CoV-2 proteins reveals Orf6 pathogenicity, subcellular localization, host interactions and attenuation by Selinexor. Cell Biosci. 2021, 11, 58. [CrossRef]

20. Zhu, J.; Lee, J.; van de Leemput, J.; Lee, H.; Han, Z. Functional analysis of SARS-CoV-2 proteins in Drosophila identifies Orf6-induced pathogenic effects with Selinexor as an effective treatment. Cell Biosci. 2021, in press. [CrossRef]

21. Chen, C.-Y.; Ping, Y.-H.; Lee, H.-C.; Chen, K.-H.; Lee, Y.-M.; Chan, Y.-J.; Lien, T.-C.; Jap, T.-S.; Lin, C.-H.; Kao, L.-S.; et al. Open reading frame $8 \mathrm{a}$ of the human severe acute respiratory syndrome coronavirus not only promotes viral replication but also induces apoptosis. J. Infect. Dis. 2007, 196, 405-415. [CrossRef] 
22. Rashid, F.; Dzakah, E.E.; Wang, H.; Tang, S. The ORF8 protein of SARS-CoV-2 induced endoplasmic reticulum stress and mediated immune evasion by antagonizing production of interferon beta. Virus Res. 2021, 296, 198350. [CrossRef]

23. Ceraolo, C.; Giorgi, F.M. Genomic variance of the 2019-nCoV coronavirus. J. Med. Virol. 2020, 92, 522-528. [CrossRef] 